\title{
Evaluación de la eficiencia técnica de las Universidades Públicas Estatales (UPE) del noroeste de México mediante Análisis Envolvente de Datos (DEA) Technical efficiency evaluation in Public State Universities (UPE) of the Mexico's northwest through Data Envelopment Analysis (DEA)
}

Daniela Alcaraz-Ochoa ${ }^{1}$ y Deyanira Bernal-Domínguez ${ }^{1}$

Palabras Clave: DEA; eficiencia técnica; financiamiento; UPE Recepción: 30-01-17 / Aceptación: 16-05-17

\section{Resumen}

El presente estudio extiende la evidencia empírica existente dentro de la Economía de la Educación al evaluar la eficiencia técnica en el uso de los recursos federales otorgados a las Universidades Públicas Estatales (UPE) mediante procesos incrementales ordinarios y fondos extraordinarios, los cuales son considerados como insuficientes por este tipo de instituciones. El objetivo de este trabajo es aplicar un procedimiento que permita conocer la eficiencia técnica alcanzada por las UPE del noroeste de México durante el periodo 2014-2015. La metodología empleada fue mediante el uso del Análisis Envolvente de Datos (DEA) en su modelo CCR-O. Se estima la función de producción utilizando las variables de financiamiento federal (ordinario y extraordinario) como input $y$ alumnos de nuevo ingreso, alumnos egresados, programas educativos acreditados y cuerpos académicos como outputs, obtenidos mediante las bases de datos de la Asociación Nacional de Universidades e Instituciones de Educación Superior (ANUIES) y la Universidad Nacional Autónoma de México (UNAM). Los hallazgos principales fueron que la relación entre las variables utilizadas como input y outputs refleja una eficiencia técnica promedio de 0.860379 (en escala de 0 a 1), siendo técnicamente eficientes la Universidad Autónoma de Baja California (UABC) y el Instituto Tecnológico de Sonora (ITSON). Se obtienen los datos de peer group y targets para su consideración como referencia de mejora de las instituciones que no resultan técnicamente eficientes.

\footnotetext{
Abstract

${ }^{1}$ Universidad Autónoma de Sinaloa. E-mail: alcarazok@gmail.com

(C) Universidad De La Salle Bajío (México)
}

The present study extends empirical evidence within the Economics of Education in evaluating the technical efficiency in the use of federal resources granted to State Public Universities (UPE) through regular incremental processes and extraordinary funds, which are considered insufficient 
by this type of institutions. The objective of this work is to apply a procedure that allows to know the technical efficiency achieved by the UPE of northwest Mexico during the period 2014-2015. The methodology used was the Data Envelopment Analysis (DEA) in its CCR-O model, the production function was estimated using the federal financing (ordinary and extraordinary) variables as input and new students, students graduated, accredited educational programs and academic bodies as outputs, obtained through the databases of the National Association of Universities and Institutions of Higher Education (ANUIES) and the National Autonomous University of Mexico (UNAM). The main findings were that the relationship between the variables used as inputs and outputs reflects an average technical efficiency of 0.860379 ( 0 to 1 scale), being the technically efficient the Autonomous University of Baja California (UABC) and the Technological Institute of Sonora (ITSON). Peer group and target data are obtained for consideration as a reference for improving institutions that are not technically efficient.

Keywords: DEA; technical efficiency; financing; UPE. 


\section{Introducción}

La relevancia de un sistema educativo eficiente ha permitido su estudio mediante líneas de investigación como la Economía de la Educación, para así extender el conocimiento entre la relación que presentan las variables de capital humano producido mediante el uso eficiente de los recursos disponibles. Estos esfuerzos se llevan a cabo al considerar que "la relación entre la eficiencia del sistema educativo y la economía son trascendentales para el bienestar social", (Cano Gamboa, 2008, 18).

Existen en México 34 Universidades Públicas Estatales (UPE) las que, según el Anuario Estadístico de Educación Superior de la ANUIES (2016), durante el periodo 2014-2015 atendieron a 1, 022,983 estudiantes de educación superior, lo que representa el $39.6 \%$ de la matrícula total atendida por las 915 Instituciones de Educación Superior (IES) con financiamiento público. En las ocho UPE ubicadas en los estados del noroeste del país (Figura 1), durante el mismo periodo se atendieron un total de 244,274 estudiantes del nivel superior (Técnico Superior Universitario y Licenciatura en modalidad escolarizada y no escolarizada). De ahí la importancia de estas instituciones en la región, por la atención del $47 \%$ del total de estudiantes universitarios, incluyendo aquellos que cursan sus estudios tanto en instituciones públicas como en privadas.

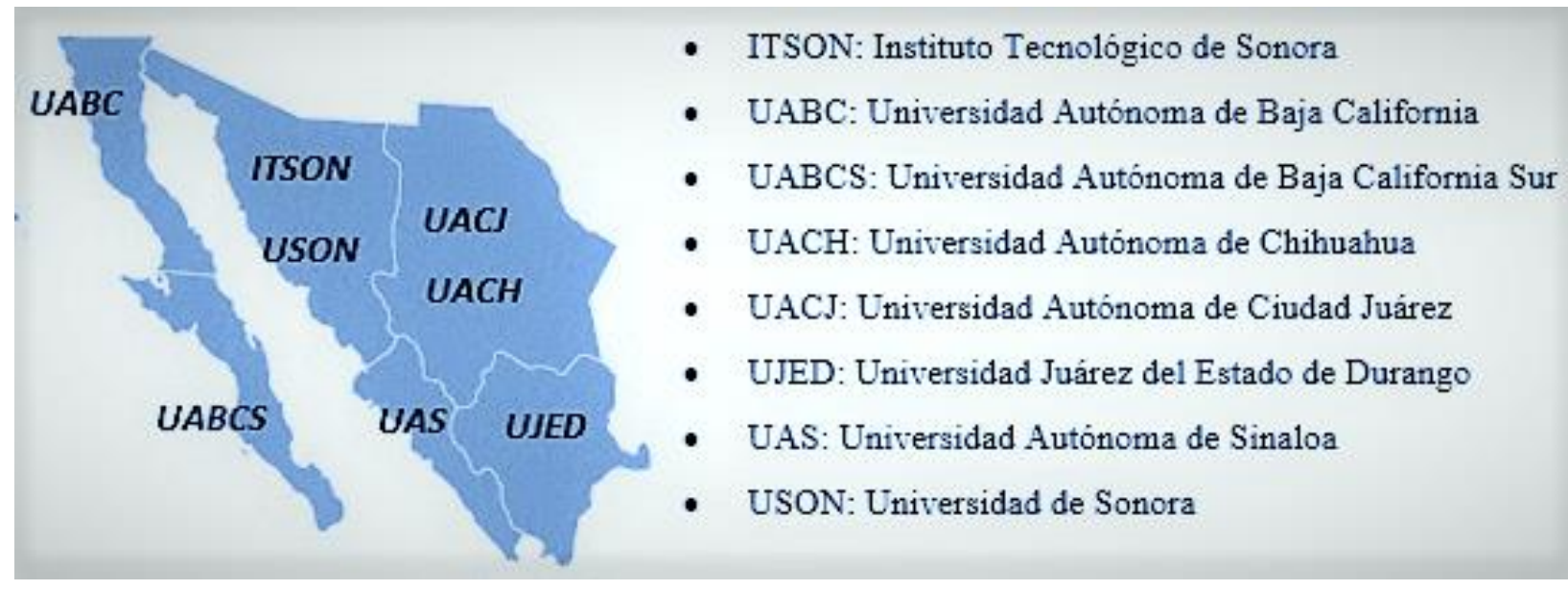

Figura 1. Universidades Públicas Estatales (UPE) del Noroeste de México.

Fuente: Elaboración propia.

A pesar de la importancia de las UPE dentro del sistema educativo del país, estas generalmente presentan dificultades financieras. Estudios como el de Güemes-Castorena (2008) afirman que las UPE no son financiadas con base en la eficiencia del desempeño de sus funciones, lo que causa 
que se presente insuficiencia de recursos en estas instituciones. Por otro lado, con base en lo publicado por la Secretaría de Educación Pública en el diagnóstico del programa de apoyos para sanaeamiento financiero y la atención a problemas estructurales de las UPE externa "La insuficiencia de recursos de las UPE ha dificultado tanto su operación adecuada como la realización de los planes de desarrollo institucional con altos estándares de calidad" (SEP, 2014).

Este escenario frecuentemente se adjudica a la política federal de financiamiento ordinario a través de incrementos porcentuales con base en un subsidio "irreductible". Lo que provoca que se presenten casos en donde las UPE reciben financiamiento por alumno debajo de la media nacional. Al paso del tiempo, esta política ha dado lugar a la implementación de fondos y programas extraordinarios ofertados mediante procesos concursales con la intención de focalizar los recursos al logro de objetivos específicos. La expansión de los servicios educativos, la atención de los sistemas de pensiones y jubilaciones y el personal de apoyo administrativo no reconocido, son algunos de los problemas estructurales que atienden este tipo de recursos.

A lo anterior hay que agregar la actual demanda social por el uso eficiente de los recursos dentro del sector público, ligada a otras corrientes como la Nueva Gestión Pública, que tiene su origen en el trabajo de Hood (1991, 5) y se centra, entre otras problemáticas, en la evaluación del desempeño y el uso eficiente de los recursos dentro del sector público "haciendo más con menos". Así pues, especialistas de la Economía de la Educación han considerado la evaluación de la eficiencia de Instituciones de Educación Superior Públicas (IESP) como un punto relevante, "especialmente por la necesidad de rendir cuentas a la sociedad sobre el empleo de los recursos públicos, así como de mejorar a nivel institucional en un entorno altamente competitivo a nivel internacional" (Fernández-Santos et al., 2015, 493).

En tanto, las interrogantes que guían este trabajo son ¿Cómo evaluar la eficiencia del uso de los recursos federales ordinarios y extraordinarios asignados a las Universidades Públicas Estatales del noroeste de México mediante el Análisis Envolvente de Datos?, ¿Cuál es el grado de eficiencia que presentan las Universidades Públicas Estatales del noroeste de México en cuanto al uso de sus recursos federales ordinarios y extraordinarios durante el periodo 2014-2015? y ¿Qué aspectos de mejora se pueden seguir para el incremento de la eficiencia de cada Universidad Pública Estatal evaluada?

Estas preguntas se establecen con el objetivo de aplicar un procedimiento que permita conocer la eficiencia técnica alcanzada por las UPE del noroeste de México durante el periodo 
indicado, así como identificar alternativas de mejora propuestas por el modelo para cada UPE que no resulte técnicamente eficiente.

La hipótesis que se plantea, afirma que las instituciones analizadas son generalmente ejecutoras eficientes de los recursos destinados para su operatividad, en una coyuntura donde su financiamiento federal es considerado insuficiente.

\section{¿Qué es eficiencia en las UPE?}

La coyuntura previamente expuesta es la razón por la que "las tareas universitarias implican que las instituciones sean gobernadas por medios eficientes y eficaces” (Hernández Bringas et al., 2015, 212). Por lo tanto, para contribuir a la evaluación de la eficiencia en el sector educativo es necesario tener una acepción de lo que se considera eficiente en este ámbito, ya que por su naturaleza presenta una problemática muy común propia del sector público: el desconocimiento de la función de producción debido a la ausencia de mercado. Una función de producción consiste en la relación existente entre los insumos o recursos (inputs) utilizados y los productos (outputs) esperados, por tanto, el uso del término de "eficiencia técnica" propuesto por Farrell $(1957,254)$ es el óptimo para referirse a esta condición dentro del ámbito de las UPE.

A su vez, la multiplicidad de sus actividades se convierte en otro obstáculo para la definición de los inputs y outputs esperados. No obstante, la revisión de la literatura realizada por Martín Rivero $(2007,805)$ identifica como parte de los inputs a los recursos financieros con los que cuenta para el desarrollo de sus funciones, mientras que los outputs están relacionados específicamente a dos ejes: docencia e investigación. El primero de estos está directamente relacionado a la formación de recursos humanos con capacidades específicas propias de adquirir en este tipo de instituciones. Por lo que se visualizan outputs tales como alumnos, egresados e incluso la carga docente. En tanto, el rubro de investigación ha cobrado fuerza como uno de los productos esperados dentro de las UPE dentro de los últimos años, un ejemplo de las variables relacionadas a este rubro son los Cuerpos Académicos.

Tomando en cuenta estos factores, un método recomendable para la evaluación de las UPE es el Análisis Envolvente de Datos (DEA), una técnica de programación matemática desarrollada en el trabajo de Charnes et al. (1978) que hace uso del análisis de frontera, útil para realizar evaluaciones de unidades objeto de estudio o Decision Making Units (DMU) con características de producción similares que desconocen su función de producción.

No 19, Vol. 9 (2), 2017. ISSN 2007 - 0705, pp.: 393 - 410 
El DEA "permite la construcción de una superfice envolvente, frontera eficiente o función de producción empírica, a partir de los datos disponibles del conjunto de unidades objeto de estudio, de forma que las unidades que determinan la envolvente son denominadas unidades eficientes", (Coll Serrano y Blasco Blasco, 2000, 18).

\section{Evidencia empírica}

Conviene destacar el previo uso del DEA en investigaciones de corte científico: Emrouznejad et al. $(2008,153)$ confirman que esta técnica se ha utilizado al menos en 1,621 trabajos científicos en diversos journals alrededor del mundo. Particularmente en el ámbito educativo, el DEA se ha aplicado en estudios de diversos países, de los más citados son McMillan y Datta (1998), quienes aplicaron el DEA para medir la eficiencia técnica en 45 universidades canadienses. Por su parte, Gómez Sancho (2001) realiza una aproximación a la evaluación de la eficiencia en 35 universidades españolas (públicas y privadas). Otro caso en España es el de Vázquez Rojas (2010) quien realizó también la evaluación a 43 universidades públicas presenciales. Más recientemente está Mikušová (2015) quien evaluó a las IES de la República Checa.

En México, también se identifican trabajos científicos que aplican el DEA en ambientes educativos, aunque con propósitos diferentes:

- Sigler (2004) aplicó el DEA para evaluar la eficiencia relativa de la producción de investigación económica durante en periodo 1990-2002 en CIDE, COLMEX, IPN, UAM y UNAM. La metodología usada en esta investigación toma en cuenta como inputs a los investigadores de estas instituciones y como outputs libros y artículos publicados por éstos. Llegó a la conclusión de que la eficiencia de estos centros públicos de investigación durante dicho periodo fue muy baja (62.6\% en promedio, en escala de 0 a $100 \%)$.

- Güemes-Castorena (2008) realizó una aplicación del DEA en 34 UPE como propuesta para referencia en la asignación de recursos y contrarrestar la problemática de los presupuestos irreductibles que no consideran el desempeño de estas instituciones. En su estudio destacó el hallazgo de la no correlación entre las variables eficiencia obtenida y recursos otorgados a las UPE.

- Altamirano-Corro y Peniche-Vera (2014) hicieron uso del DEA en combinación de Redes Neuronales Artificiales (RNA) para la evaluación de la eficiencia institucional en 51 escuelas de ingeniería utilizando los indicadores del Programa Integral de Fortalecimiento

No 19, Vol. 9 (2), 2017. ISSN 2007 - 0705, pp.: 393 - 410 
Institucional (PIFI 2008-2009). La combinación de ambas técnicas y el uso de los indicadores para la obtención de resultados se consideraron satisfactorios.

- Navarro Chávez et al. (2016) midieron la eficiencia de 32 universidades públicas en México durante 2012 con el uso del DEA, en conjunto con la técnica del bootstrap para darle robustez a los cálculos. El input utilizado en este trabajo fue el financiamiento y los outputs aplicados fueron variables relativas a la enseñanza e investigación (matrícula y graduados totales en licenciatura y posgrado, número de docentes, número de docentes con grado de doctor, número de miembros en el Sistema Nacional de Investigadores y número de publicaciones de artículos registrados en el índice ISI-Thomson). Obtuvieron una eficiencia promedio de 0.71 (en escala de 0 a 1 ) en un modelo con rendimientos constantes a escala.

El análisis de la evidencia empírica demuestra la relevancia que tiene para múltiples investigadores el estudio de la eficiencia en las instituciones educativas, así como la aptitud del uso del DEA en este ámbito. Además, se observa que aun cuando hay indicadores generalmente aceptados para conformar la función de producción, el uso de éstos puede ser tomado con discrecionalidad para utilizarse como inputs y outputs dirigidos a cumplir con los propósitos específicos que cada investigación proyecte.

\section{Metodología}

Se realizó la evaluación de la eficiencia técnica de las ocho UPE ubicadas en el noroeste de México durante el periodo 2014-2015, aplicando el DEA en su modelo CCR con orientación a output (CCR-O), desarrollado por Charnes et al. $(1978,430)$. El modelo considera que las unidades de estudio se encuentran en niveles constantes de escala, ya que este modelo busca, dado un nivel de inputs, el máximo incremento proporcional de los outputs (Coll Serrano y Blasco Blasco, 2000, 21). 


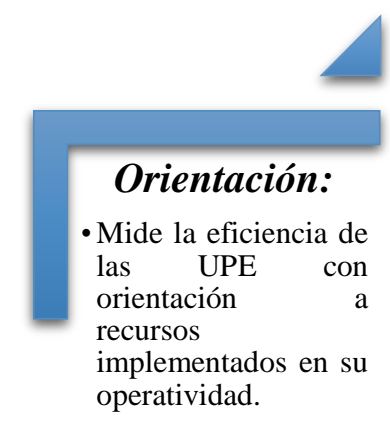

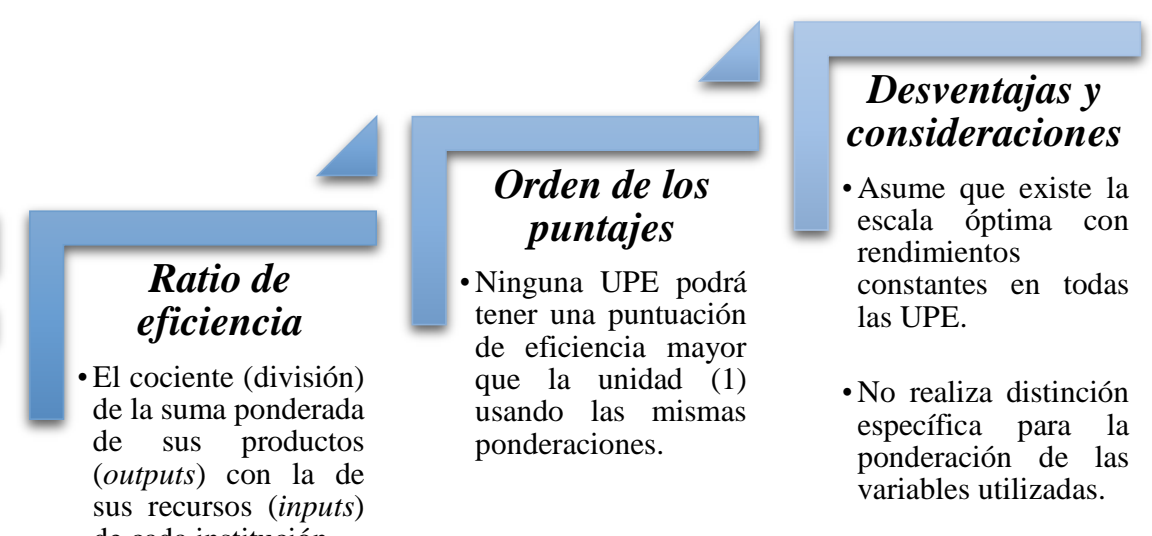

Figura 2. Características del modelo DEA CCR-O en el entorno de las UPE.

Fuente: Elaboración propia.

Con el propósito de mitigar las desventajas que en la figura anterior se observan, se hizo una selección de variables relacionadas de manera general con los indicadores de desempeño deseables por las IES que ejecutan los recursos. Algunas de las propiedades que motivaron el uso del DEA es el ofrecer información individualizada de las UPE por medio de una puntuación de eficiencia técnica, relacionar simultáneamente todos los recursos con su producción y el que no sea necesario que las variables estén expresadas en una misma unidad de medida. El modelo general CCR-O se representa de la siguiente manera:

$$
\begin{gathered}
\max z=\sum_{j=1}^{J} v_{j m} y_{j m} \\
\text { Sujeto a } \\
\sum_{i=1}^{I} u_{i m} x_{i m}=1 \\
\sum_{j=1}^{J} v_{j m} y_{j n}-\sum_{i=1}^{I} u_{i m} x_{i n} \leq 0 ; n=1,2, K, N \\
v_{j m}, u_{i m} \geq \varepsilon ; i=1,2, K, I ; \quad j=1,2, K, J
\end{gathered}
$$

Donde $z$ es la eficiencia de la $m$-ésima DMU 


$$
\begin{gathered}
y_{j m} \text { es el } j \text {-ésimo output de la } m \text {-ésima DMU } \\
v_{j m} \text { es el peso de ese output } \\
x_{i m} \text { es el } i \text {-ésimo input de la } m \text {-ésima DMU } \\
u_{i m} \text { es el peso de ese input, y } \\
y_{j n} \text { y } x_{i n} \text { son el } j \text {-ésimo } \text { output y } i \text {-ésimo input, respectivamente, de la } n \text {-ésima } \\
\text { DMU, } n=1,2, \ldots, N .
\end{gathered}
$$

Fuente: Ramanathan $(2003,45)$.

En tanto que la presente evaluación hace uso de cinco variables (un input y cuatro outputs), la función del modelo se representa como matriz transpuesta de los datos de la siguiente manera:

$$
v_{11} * y_{12}+v_{21} * y_{22}+v_{31} * y_{32}+v_{41} * y_{42}-u_{11} * x_{12} \leq 0
$$

En cuanto a la selección de las variables, se toma como única variable de tipo input, a los recursos ordinarios y extraordinarios asignados por la federación a las UPE seleccionadas durante el periodo indicado, esto significa que el análisis busca identificar aquellas UPE que maximizan el uso de sus recursos financieros para producir sus outputs.

Las variables consideradas como outputs para el modelo hacen referencia tanto a los estudios previos existentes, como a los indicadores considerados en las fichas técnicas para evaluación al desempeño a nivel propósito de los programas presupuestarios ordinarios y extraordinarios de origen federal (SEP, 2016). De esta manera las variables y los datos recopilados a evaluar se concentran en la siguiente tabla. 
Tabla 1. Selección de inputs y outputs.

\begin{tabular}{|c|c|c|c|c|c|}
\hline & Inputs & Outputs & & & \\
\hline UPE & $\begin{array}{l}\text { FIN } \\
\text { Financiamiento } \\
\text { Federal Ordinario y } \\
\text { Extraordinario* } \\
\text { (Millones de pesos) }\end{array}$ & $\begin{array}{l}\text { ANI } \\
\text { Alumnos } \\
\text { nuevo } \\
\text { ingreso } \\
\text { (nivel } \\
\text { superior) }\end{array}$ & $\begin{array}{l}A E \\
\text { Alumnos } \\
\text { Egresados } \\
\text { (nivel } \\
\text { superior) }\end{array}$ & $\begin{array}{l}\text { PEA } \\
\text { Programas } \\
\text { Educativos } \\
\text { Acreditados } \\
\% \\
\text { Institucional }\end{array}$ & $\begin{array}{l}\text { Cuerpos } \\
\text { Académicos }\end{array}$ \\
\hline ITSON & 566.93 & 3,721 & 1,857 & 32.7 & 39 \\
\hline UABC & $1,629.45$ & 10,090 & 7,930 & 52.3 & 175 \\
\hline UABCS & 404.24 & 1,705 & 496 & 20 & 20 \\
\hline UACH & 956.35 & 5,582 & 3,780 & 32.7 & 54 \\
\hline UACJ & $1,092.22$ & 5,540 & 2,448 & 40.9 & 64 \\
\hline UJED & $1,206.20$ & 2,231 & 2,330 & 41.7 & 48 \\
\hline UAS & $3,735.43$ & 20,055 & 9,759 & 37.4 & 101 \\
\hline USON & $1,112.63$ & 6,511 & 2,989 & 42 & 84 \\
\hline
\end{tabular}

* Incluyen: Subsidios Federales para Organismos Descentralizados Estatales. Programa de Expansión en la Oferta Educativa en Educación Media Superior y Superior (PROEXOEES). Programa de Fortalecimiento de la Calidad en Instituciones Educativas (PROFOCIE) (antes PIFI). Programa para el Desarrollo Profesional Docente, para el Tipo Superior (PRODEP) (antes PROMEP).

Fuente: Elaboración propia con datos de UNAM (2016) y ANUIES (2016).

Los datos de las variables han sido recolectados del portal de ExECUM, un explorador de datos del Estudio Comparativo de Universidades Mexicanas (ECUM) de la Universidad Nacional Autónoma de México (UNAM). De igual manera, también se recolectaron datos del Anuario 
Estadístico de Educación Superior de la Asociación Nacional de Universidades e Instituciones de Educación Superior (ANUIES). El programa ejecutado para la evaluación de los datos es el software Frontier Analyst (2016).

\section{Resultados}

Los puntajes obtenidos mediante el DEA sobre la eficiencia técnica de cada UPE son mostrados en la siguiente tabla.

Tabla 2. Resultados de evaluación de eficiencia técnica de las UPE del noroeste de México.

\begin{tabular}{|l|l|l|}
\hline UPE & EFICIENCIA & $\begin{array}{l}\text { EFICIENCIA } \\
\text { TÉCEICA }\end{array}$ \\
& & PEROMEDIO \\
\hline ITSON & 1 & \\
\hline UABC & 1 & \\
\hline UABCS & 0.857773 & \\
\hline UACH & 0.922384 & 0.860379 \\
\hline UACJ & 0.775067 & \\
\hline UJED & 0.599375 & \\
\hline UAS & 0.817997 & \\
\hline USON & 0.910439 & \\
\hline
\end{tabular}

Fuente: Elaboración propia.

Las UPE con una evaluación de 1 son aquellas que bajo el modelo aplicado, están consideradas como "técnicamente eficientes", y por lo tanto, construyen la función de producción empírica. Sin embargo en la opinión de Cooper et al. (2007) un valor de eficiencia aceptable es >0.8. De ahí que también se consideren la UABCS, UACH, UAS y USON eficientemente aceptables. Se muestra 
en la Tabla 2 una eficiencia técnica promedio de las UPE del noroeste de México de 0.860379. La tabla 3 presenta el conjunto de referencia o peer group para cada UPE con valor $<1$.

Tabla 3. Peer group para cada UPE.

\begin{tabular}{|ll}
\hline UPE & PEER GROUP \\
\hline UABCS & ITSON. \\
\hline UACH & ITSON, UABC. \\
\hline UACJ & ITSON, UABC. \\
\hline UJED & ITSON. \\
\hline UAS & ITSON. \\
\hline USON & ITSON, UABC. \\
\hline
\end{tabular}

Fuente: Elaboración propia.

Un peer group es un conjunto de referencia eficiente para las UPE que no trazan la frontera eficiente, por lo que el DEA le asigna a cada unidad un conjunto de referencia que deberá de "seguir" o "aprender" en sus prácticas para así aumentar su eficiencia técnica. Este dato constituye una referencia para la mejora de las UPE con evaluación < 1. El peer group que se le asigne tendrá la característica de contar con una combinación similar de inputs y outputs.

El DEA también hace posible el cálculo de proyecciones o targets, es decir, determina para cada UPE una aproximación unidades de output necesarias para llegar a la condición de eficiencia técnica, esto sin alterar el nivel de financiamiento, ya que como se menciona el input de financiamiento no es un valor controlable por las UPE. 
Tabla 4. Determinación de proyecciones (targets) para cada output.

\begin{tabular}{|lllll} 
UPE & ANI & AE & PEA & CA \\
& & & $(\%)$ & \\
\hline ITSON & 3721 & 1857 & 32.7 & 39 \\
\hline UABC & 10090 & 7930 & 52.3 & 175 \\
\hline UABCS & 2653 & 1324 & 23.3 & 28 \\
\hline UACH & 6052 & 4098 & 39.6 & 89 \\
\hline UACJ & 7148 & 3887 & 58.8 & 83 \\
\hline UJED & 7917 & 3951 & 69.6 & 83 \\
\hline UAS & 24517 & 12236 & 215 & 257 \\
\hline USON & 7151 & 4292 & 53.8 & 92 \\
\hline
\end{tabular}

Fuente: Elaboración propia.

La información que se muestra en la Tabla 4 funciona comúnmente para establecer metas y realizar prácticas de benchmarking, si así se desea. No obstante, hay que tener discreción para su interpretación. Un ejemplo son las proyecciones propuestas para la variable de Programas Educativos Acreditados (PEA), debido a que esta variable se encuentra expresada en porcentaje, e incluso cuando el DEA admite que las variables se encuentren expresadas en diferentes unidad de medida, sus proyecciones pueden ser objeto de confusiones. Un ejemplo de esto se observa en la variable PEA de la UAS, la cual se deberá interpretar como una sugerencia de incremento en el número de PEA a una cantidad que refleje el porcentaje recomendado.

\section{Discusión}

Tanto esta investigación como la realizada por Navarro Chávez et al. (2016), coinciden en evaluar como técnicamente eficiente, bajo rendimientos constantes, a la Universidad Autónoma de Baja California (UABC). Además, este trabajo evalúa como estrechamente cercano a la eficiencia técnica, al Instituto Tecnológico de Sonora (ITSON), con una puntuación de 0.9895. Esta 
coincidencia sucede durante los periodos de evaluación (2012 y 2014), utilizando sólo un mismo output en común (alumnos egresados). Por tanto, se puede apreciar como un indicio de que estas UPE son técnicamente eficientes en el uso de sus recursos, incluso en ámbitos no considerados por las variables de este estudio.

Se identificaron áreas de oportunidad en la aplicación del DEA en el ámbito de las UPE debido a la posibilidad del uso de esta técnica de manera discrecional para obtener información relacionada con áreas de interés categóricas, inclusive en su uso para la evaluación de las UPE de manera individual comparando áreas o departamentos internos. Es también una alternativa para futuras investigaciones, la evaluación de la totalidad de las UPE del país, para de esta manera corroborar si las UPE que forman la frontera de producción en el noroeste permanecen técnicamente eficientes, considerando otras instituciones; así sería posible la generalización de resultados a nivel nacional.

Es importante considerar algunos aspectos para contextualizar los resultados de la presente evaluación, pues incluso cuando las evaluaciones se realizan bajo los parámetros establecidos por el modelo, la selección de unas variables por encima de otras hace que los resultados se expresen bajo ciertas condiciones. La limitación del uso de variables atiende a una restricción que posee el DEA, ya que como lo mencionan Coll Serrano y Blasco $(2000,25)$ "el DEA probablemente trabaje mejor cuando el número de observaciones es aproximadamente el doble de la suma de los inputs y outputs".

Este trabajo es el primero en centrar la atención de manera particular a las UPE del noroeste de México, instituciones con una labor fundamental en el ámbito educativo del país. Por tanto, la importancia de sus hallazgos también radica en ser un referente para futuros estudios en la región.

\section{Conclusiones}

A manera de conclusión, el uso del DEA en su modelo CCR-O al incorporar las variables financiamiento, alumnos de nuevo ingreso, alumnos egresados, porcentaje de programas educativos acreditados y cuerpos académicos, concibe datos significativos respecto al desempeño de las UPE ubicadas en el noroeste de México. La relación de las variables utilizadas como inputs y outputs refleja una eficiencia técnica promedio de 0.860379 , la cual funge como evidencia empírica sobre el manejo de los recursos en estas instituciones, que durante el periodo 2014-2015 la evaluación considera como técnicamente eficiente a UABC e ITSON, y otorga un valor de

No 19, Vol. 9 (2), 2017. ISSN 2007 - 0705, pp.: 393 - 410

- 406 - 
eficiencia aceptable a UABCS, UACH, UAS y USON, según el criterio establecido por Cooper et al. (2007).

La información que tanto los peer groups como los targets aportan a cada UPE que no conforma la función de producción, son datos útiles que éstas instituciones pueden tomar en cuenta para la mejora de su eficiencia técnica, tales como: el aumento de alumnos de nuevo ingreso, alumnos egresados, porcentaje de programas educativos acreditados y cuerpos académicos.

Se comprueba la hipótesis planteada que externa que las instituciones analizadas son generalmente ejecutoras eficientes de los recursos destinados para su operatividad, debido a que seis de las ocho UPE evaluadas resultaron con un valor de eficiencia aceptable según el parámetro de Cooper et al. (2007), en una coyuntura donde su financiamiento federal es considerado insuficiente.

\section{Referencias}

Altamirano-Corro, Antonio y Peniche-Vera, Rebeca del Rocío. (2014). Metodología AED-RNA para la estimación de la eficiencia institucional: El caso de las dependencias de educación superior (DES) de ingeniería de México. Nova Scientia, 6(12), 356-378.

Asociación Nacional de Universidades e Instituciones de Educación Superior (ANUIES). (2016). Anuarios Estadísticos de Educación Superior. http://www.anuies.mx/informacion-yservicios/informacion-estadistica-de-educacion-superior/anuario-estadistico-deeducacion-superior (28 de octubre de 2016).

Cano Gamboa, Carlos Andrés. (2008). Determinantes en la eficiencia en la producción de educación. Revista de la Educación Superior XXXVII (3) (147): 17-30.

Charnes, A., Cooper, W. W., y Rhodes, E. (1978). Measuring the efficiency of decision making units. European Journal of Operational Research, 2(6), 429-444. doi: http://dx.doi.org/10.1016/0377-2217(78)90138-8 
Evaluación de la eficiencia técnica de las Universidades Públicas Estatales (UPE) del noroeste de México mediante Análisis Envolvente de Datos (DEA)

Coll Serrano, Vicente, y Blasco Blasco, Olga Ma. (2000). Evaluación de la eficiencia mediante el Análisis Envolvente de Datos: Introducción a los modelos básicos. Madrid: Universidad de Valencia.

Cooper, William W.; Seiford, Lawrence M. y Tone, Kaoru. (2007). Data Envelopment Analysis. New York: Springer.

Emrouznejad, Ali, Parker, Barnett R. y Tavares, Gabriel. (2008). Evaluation of research in efficiency and productivity: A survey and analysis of the first 30 years of scholarly literature in DEA. Journal of Socio-Economics Planning Science, 42(3), 151-157.

Farrell, M. J. (1957). The Measurement of Productive Efficiency. Journal of The Royal Statistical Society, Vol. 120(3), 253-290.

Fernández-Santos, Yolanda, Martínez-Campillo, Almudena y Fernández-Fernández, José Miguel. (2015). Determinantes de la eficiencia en las universidades públicas españolas: un análisis DEA en dos etapas. En Investigaciones de Economía de la Educación, compilado por Marta Rahona López y Jennifer Gravez, (1 ed., Vol. 10, pp. 491-508). Madrid: Asociación de Economía de la Educación.

Frontier Analyst. (2016). https://banxia.com/frontier/ (24 de noviembre de 2016).

Gómez Sancho, José María. (2001). La evaluación de la eficiencia en las universidades públicas españolas X Jornadas de la Asociación de la Economía de la Educación (411-434). Murcia, España: Asociación de la Economía de la Educación.

Güemes-Castorena, David. (2008). A DEA Decision Making Model for Higher Education Funding: VDM Verlag, SBN-10: 3639090004; ISBN-13: 978-3639090000; November 13, 2008 .

No 19, Vol. 9 (2), 2017. ISSN 2007 - 0705, pp.: 393 - 410 
Hernández Bringas, Héctor Hiram, Martuscelli Quintana, Jaime, Moctezuma Navarro, David, Muñoz García, Humberto y Narro Robles, José. (2015). Los desafíos de las universidades de América Latina y el Caribe ¿Qué somos y a dónde vamos? Perfiles Educativos IISUEUNAM, XXXVII (147), 202-218.

Hood, Christopher. (1991). A public management for all seasons? Public Administration, 69, 3-19. doi: 10.1111/j.1467-9299.1991.tb00779.x

Martín Rivero, Raquel. (2007). La eficiencia Productiva en el Ámbito Universitario: Aspectos Claves para su Evaluación. Estudios de Economía aplicada, 25(3), 793-811.

McMillan, Melville L. y Datta, Debasish. (1998). The relative efficiencies of Canadian Universities: A DEA perspective. Canadian Public Policy-Analyse De Politiques, 24(4), 485-511. doi: 10.2307/3552021

Mikušová, Pavla. (2015). An Application of DEA Methodology in Efficiency Measurement of the Czech Public Universities. Procedia Economics and Finance, 25, 569-578. doi: http://dx.doi.org/10.1016/S2212-5671(15)00771-6

Navarro Chávez, José César Lenin, Gómez Monge, Rodrigo, y Torres Hernández, Zacarías. (2016). Las universidades en México: una medida de su eficiencia a través del análisis de la envolvente de datos con bootstrap. Acta Universitaria, 26(6), 49-58. doi: 10.15174/au.2016.911

Ramanathan, Ramu. (2003). An Introduction to data envelopment analysis: A tool performance measurement. New Delhi: Sage publications.

Secretaría de Educación Pública (SEP). (2016). Acciones y Programas 2014-2015. http://www.gob.mx/sep/acciones-y-programas/2014-2015-diseno?state=published $(18 \mathrm{de}$ octubre de 2016). 
Secretaría de Educación Pública (SEP). (2014). Diagnóstico U081 Apoyos para saneamiento financiero y la atención a problemas estructurales de las UPES. https://www.gob.mx/cms/uploads/attachment/file/50159/Diagno_stico.pdf (18 de octubre de 2016.

Sigler Merchand, Luis Ángel. (2004). Aplicación del Data Envelopment Analysis a la producción de investigación económica en la Ciudad de México: la eficiencia relativa del CIDE, COLMEX, IPN, UAM y UNAM (1990-2002). Ponencia presentada en 4th International Symposium of Data Envelopment Analysis and Performance Management, Birmingham.

Universidad Nacional Autónoma de México. (2016). Explorador de datos del Estudio Comparativo de Universidades Mexicanas (ExECUM), http://www.execum.unam.mx/ (28 de ocutubre de 2016).

Vázquez Rojas, Angélica María. (2010). Estudio sobre la eficiencia técnica de las universidades públicas presenciales españolas. En Investigaciones de Economía de la Educación, compilado por María Jesús Mancebón Torrubia, Domingo Pérez Ximénez-de-Embún y José María Gómez Sancho y Gregorio Giménez Esteban, (Vol. 5, pp. 689-702). Zaragoza: Asociación de Economía de la Educación. 